hospital, modified Glasgow scale at 1 month and 3 months following discharge from hospital.

Results: Children had a mean age of 4.7 years, and $40 \%$ were females. The fatality rate was $34 \%$. Age and gender were similar between groups $(p>0.2)$. Survival was independently predicted by GCS score at admission to ICU $(p<0.05)$.

Conclusions: Multiple factors may influence outcome in pediatric TBI, and heterogenicity of the population is one of the difficulties in evaluating them. Evaluating outcome and identifying predictors of poor outcome help clinicians in making decisions during the acute phase of treatment.

\section{A0015 Assessment of Dynamic Predictors of Fluid Responsiveness in Patients Undergoing Supratentorial Neurosurgery Using Transesophageal Echocardiography Ajay P. Hrishi, ${ }^{1}$ Manikandan Sethuraman, ${ }^{1}$ Girish Menon ${ }^{2}$ ${ }^{1}$ Division of Neuroanesthesia, Sree Chitra Tirunal Institute for Medical Sciences and Technology, Trivandrum, Kerala, India ${ }^{2}$ Department of Neurosurgery, Kasturba Medical College Manipal, India}

Background: Patients presenting for neurosurgery are at risk of hypovolemia due to ongoing osmotherapy, neuroendocrine responses, nausea, and vomiting. Fluid therapy is frequently used in neurosurgical patients to improve hemodynamics. Only 40 to $70 \%$ of patients respond to volume expansion. The aim of this study was to assess the utility of newer dynamic predictors of fluid responsiveness such as delta down (DD), superior vena cava collapsibility index (SVCCI), and aortic velocity time integral variability (VTIAoV) in patients undergoing neurosurgery.

Materials and Methods: Thirty patients undergoing neurosurgery for supratentorial craniotomy and excision were enrolled in this prospective study. Post-induction, vitals, anesthetic parameters, and study variables were recorded as the baseline. Following this, patients received a fluid bolus of $10 \mathrm{~mL} / \mathrm{kg}$ of colloid over 20 minutes, and measurements were repeated post loading. Data were expressed as mean \pm SD. Comparison of normally distributed continuous variables was evaluated with Student's t-test, and $p<0.05$ was considered as statistically significant. The predictive ability of variables for fluid responsiveness was determined using Pearson's coefficient analysis ( $r$ ).

Results: There were 22 volume responders and 8 nonresponders. $\mathrm{DD}>5 \mathrm{~mm} \mathrm{Hg}$ was efficient in differentiating the responders from nonresponders $(p<0.05)$ with a sensitivity and specificity of $90 \%$ and $85 \%$, respectively, with a predictive ability, $r=0.716$. SVCCI of $>38 \%$ was $100 \%$ sensitive and $95 \%$ specific in detecting the volume status and in differentiating the responders from nonresponders $(p<0.05)$ with an excellent predictability, $r=0.906$. VTIAoV $>20 \%$ too proved to be a good predictor, with a sensitivity and specificity of $100 \%$ and $90 \%$, respectively, with a predictive power, $r=0.732$.
Conclusions: Our study showed that $73.33 \%$ of patients presenting for intracranial surgeries were fluid responders despite static indices of hemodynamics like heart rate, blood pressure, and CVP being within the normal range. Among the variables, SVCCI $>38 \%$ is an excellent predictor followed by VTIAoV > 20\% and DD > $5 \mathrm{~mm} \mathrm{Hg}$ in assessing the fluid status in this population.

\section{A0016 Infection in Neurosurgical Intensive Care Unit: A 3-Year Study \\ Deepa Chaturvedi, ${ }^{1}$ Pragnya P. Jena, ${ }^{2}$ Kavita Sandhu, Bansidhar Tarai ${ }^{2}$ \\ ${ }^{1}$ Department of Anaesthesia, Max Super Speciality Hospital, Saket, New Delhi, India \\ 2Department of Microbiology, Max Super Speciality Hospital, Saket, New Delhi, India}

Background: In the neurosurgical and neurocritical patient community, infection rate depends on the severity of illness at the time of presentation such as Glasgow coma score, associated comorbidities, and the exposure to invasive devices such as endotracheal tube, central venous catheters, and urinary catheters. In addition, neuroscience-specific devices, such as ventricular/lumbar catheters, also increase chances of infection. There is paucity of quality data about infection rate in neurosurgical ICU (NSICU) in India. We share our experience from a dedicated NSICU in north India.

Materials and Methods: This was a retrospective and observational study in NSICU. All patients who were admitted to NSICU, whose infection fulfilled the CDC (Centers for Disease Control and Prevention)/National Healthcare Safety Network (NHSN) criteria, were included in the study.

Results: A total of 229 patients were included in our study. Maximum percentage of patients admitted directly from home was $41.2 \%$. Length of stay (LOS) in the ICU ranged from 2 to 60 days. Average LOS in ICU was 6 days. A comprehensive of 57 laboratory-confirmed infections were identified in 53 patients, representing a prevalence rate of $23 \%$ and an incidence rate of 20.3/1,000 patient-days. The site-specific frequency of infections was: 35 urinary tract infections, 15 bloodstream infections, 2 SSI, single case of pneumonia. Various risk factors associated with development of HAIs were antibiotics use before ICU admission ( $p<0.001$, OR=6.775), presence of devices such as endotracheal tube $(p<0.001$, OR $=7.450)$, urinary catheter $(p=0.002, \mathrm{OR}=2.386)$, central line $(p<0.001, \mathrm{OR}=8.022)$, location before admission $(p=0.038$, $\mathrm{OR}=2.063)$, ICU stay $>7$ days $(p=0.003, \mathrm{OR}=2.724)$, and malignancy $(p=0.001, \mathrm{OR}=8.775)$.

Conclusions: Neurosurgical patients are particularly vulnerable to infection because of the formidable nature of their illness, the frequency of associated trauma, and the presence of invasive devices. This could be the reason for high prevalence rate of infection in these patients, which can be avoided by treating the patients in a dedicated NSICU with strict infection control protocol. Nonetheless, in our study we observed low infection rate in neurosurgical patients. 